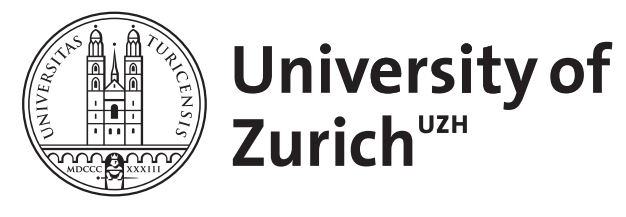

\title{
Extraversion, alcohol, and enjoyment
}

Ruch, Willibald

\begin{abstract}
The present experiment examines the effects of extraversion and consumption of alcohol on facial and verbal indicators of humor-induced positive affect. Sixty one female students were placed into one of three alcohol conditions (no ethanol, low dose, high dose) and were exposed to 30 slides containing jokes or cartoons. Facial measurements of smiling and laughter, funniness and aversiveness of the humorous stimuli, and mood dimensions served as dependent variables. Extraversion predicted both the frequency and intensity of facial exhilaration. Extraversion also moderated the effects of alcohol. Extraverts showed less verbal and facial exhilaration in the high alcohol condition than in the no alcohol condition. A low dose of alcohol increased the ambiverts' facial enjoyment but-contrary to the expectations based on the Eysenckian drug postulate-the introverts' verbal and facial behavior was not affected by alcohol consumption. The effects of extraversion and alcohol on humor-induced exhilaration seemed to be mediated by differences in positive affectivity. The verification of most of the tested hypotheses was contingent on the separation of enjoyment and non-enjoyment displays.
\end{abstract}

DOI: https://doi.org/10.1016/0191-8869(94)90113-9

Posted at the Zurich Open Repository and Archive, University of Zurich ZORA URL: https://doi.org/10.5167/uzh-77508

Journal Article

Originally published at:

Ruch, Willibald (1994). Extraversion, alcohol, and enjoyment. Personality and Individual Differences, 16(1):89-102.

DOI: https://doi.org/10.1016/0191-8869(94)90113-9 
This manuscript was published as:

Ruch, W. (1994). Extraversion, alcohol, and enjoyment. Personality and Individual Differences, 16, 89-102. 
Extraversion, alcohol, and enjoyment - 1 -

Extraversion, alcohol, and enjoyment.

\section{WILLIBALD RUCH}

Department of Physiological Psychology

University of Düsseldorf

March 1993

Running Head: Extraversion, alcohol, and enjoyment

Authors address:

PD Dr. Willibald Ruch, Department of Physiological Psychology, University of Düsseldorf, Universitätsstraße 1, 4000 Düsseldorf, Germany

Phone: office: 001149 (211) 311-3067 (Secretary: -2062) $\quad$ home: 001149 (211) 376363

FAX: 001149 (211) 311-2856 e-mail: wruch at ze8.rz.uni-duesseldorf.de 
Extraversion, alcohol, and enjoyment - 2 -

Summary - The present experiment examines the effects of extraversion and consumption of alcohol on facial and verbal indicators of humor-induced positive affect. 61 female students were placed into one of three alcohol conditions (no ethanol, low dose, high dose) and were exposed to 30 slides containing jokes or cartoons. Facial measurements of smiling and laughter, funniness and aversiveness of the humorous stimuli, and mood dimensions served as dependent variables. Extraversion predicted both the frequency and intensity of facial exhilaration. Extraversion also moderated the effects of alcohol. Extraverts showed less verbal and facial exhilaration in the high alcohol condition than in the no alcohol condition. A low dose of alcohol increased the ambiverts' facial enjoyment but--contrary to the expectations based on the Eysenckian drug postulate--the introverts' verbal and facial behavior was not affected by alcohol consumption. The effects of extraversion and alcohol on humor-induced exhilaration seemed to be mediated by differences in positive affectivity. The verification of most of the tested hypotheses was contingent on the separation of enjoyment and non-enjoyment displays. 
Extraversion, alcohol, and enjoyment - 3 -

\section{INTRODUCTION}

The Eysenckian extraversion superfactor of personality is considered to represent a general disposition for positive affect (Eysenck \& Eysenck, 1985). In regards to mood states, extraverts are expected to show variation between positive affect and neutrality whereas the mood states of high $\mathrm{N}$ scorers are expected to vary predominantly between negative affect and neutrality. This hypothesis received support from a variety of studies on mood swings and experimentally induced mood (e.g., Costa \& McCrae,1980; Hepburn \& Eysenck, 1989; Larsen \& Ketelaar, 1989).

As regards smiling and laughter, the behavioral indicators of positive affect, the role of extraversion is less clear. There is no study which explicitly tested the postulate that extraverts have a tendency to "laugh and be merry" (Eysenck \& Eysenck, 1975, p. 9). However, correlations between smiling/laughter and extraversion were reported in a few studies (see Ruch, 1990 and Ruch \& Deckers, in press, for reviews) and these results did not provide much support for the hypothesis. The reasons why these studies failed to verify the hypothesis of a relationship between extraversion and positive affect may be manifold. For example, the assessment of smiling/laughter could lack sophistication or there was no control of the nature of the emotion accompanying the smile or laugh. This is especially the case when smiles or laughs were displayed during a conversation; here the type of associated emotion can vary and they also can represent social signals rather than emotional displays.

Another reason for this lack of findings could have resulted from the failure to discriminate among different types of smiles/laughs according to their morphology. For example, despite a sophisticated assessment of laughter and the use of a homogeneous amusing stimulus, Shimizu, Kawasaki, Azuma, Kawasaki, and Nishimura (1982) did not find a relationship between their "laughing score" and extraversion as assessed by the Maudsley Personality Inventory. In this study of 34 normals and 8 schizophrenic patients, Shimizu et al. (1982) recorded the amount of smiling/laughter induced by a humorous tape on a 0 to 5 step "laughing score." The activity of the zygomatic major (the main smiling/laughing muscle) formed the core of this laughing score. The 
EMG amplitude was broken down into three intervals yielding 1,2, or three points respectively. Further points were scored if there were either changes of at least one of the three indicators, i.e., GSR, plethysmogram, and respiration, or if body movement and/or phonation occurred. However, all zygomatic activity entered the laughing score, whether or not it was related to amusement. No separation of smiles/laughs reflecting enjoyment of the presented material and other types of smiles/laughs were undertaken. One could argue that the failure to verify the extraversion-laughter relationship in the Shimizu et al. (1982) study might have resulted from the presence of non-enjoyment smiles, which suppressed the expected effects.

\section{Differentiation among Smiles and Laughter}

Due to the work of Ekman, Friesen, and associates (e.g., Ekman, 1985; Ekman \& Friesen, 1982) our understanding of different types of smiles is far advanced. Whereas five muscles (zygomatic major, zygomatic minor, risorius, levator anguli oris, buccinator) insert in the lip corner area and are able to create movements of the lip corners which are interpretable as "smiles," only one of them (the zygomatic major) is active during positive affect. The sole action of this muscle, however, is not sufficient for the creation of a smile of enjoyment. As early as 1862, the French anatomist Duchenne du Bologne noted that smiles generated without the involvement of the orbicularis oculi muscle appear to be faked or false. After being neglected for more than a century this hypothesis was picked up again in the last decade and is now substantiated by several studies (for a review, see Ekman, Davidson \& Friesen, 1990). Thus, a genuine smile--as displayed during positive affect--is created by the joint action of the zygomatic major muscle and the lateral part of the orbicularis oculi muscle; the former pulls the lip corner obliquely up and back and deepens the furrow running from the nostril to the lip corner. The latter lifts the cheeks upward and draws the skin toward the eyes from the temple and cheeks; furthermore it narrows the eye opening and may cause crow's feet wrinkles to appear at the outer corner of the eye opening (Ekman \& Friesen, 1982). 
However, the presence of these two muscles is not a sufficient condition for an enjoyment smile. It is necessary to distinguish between spontaneous (or emotional) and voluntary facial movements as they occur in felt and false smiles, respectively. Individuals may want to convince somebody that they experience a positive affect although nothing much is felt or even negative emotions are present. Such false smiles usually are created without involvement of the orbicularis oculi muscle, however, their identification is not solely dependent on this so-called Duchenne marker (Frank \& Ekman, 1993). Voluntary smiles are more frequently unilateral (present in one half of the face only) or asymmetrical (stronger in one half of the face); their onset is abrupt or otherwise irregular but not smooth; they are more frequently too short (less than half of a second) or too long (more than 4 seconds); and they are more frequently asynchronous (i.e., the zygomatic major and orbicularis oculi muscles reach their apex not at the same time). The validity of these markers for the distinction between enjoyment smiles and false smiles are discussed by Frank and Ekman (1993).

No such differentiation has been achieved for laughter yet. Types of laughter are usually differentiated by the emotion attributed to it but not on a morphological basis. However, the contraction of the zygomatic major and the orbicularis oculi muscles also forms the core of the laughter of positive affect (Ruch, 1990). Several additional muscles are involved in laughing, but their exact number is not yet known (Ruch, 1993). They might be secondary in importance because they largely serve purposes like facilitating the expulsion of air (e.g., the opening of the mouth, throwing backward the head). Also, it is likely that most of the markers distinguishing between voluntary and emotional facial movements will apply to laughs as well. For example, laughter displayed in response to humor has been shown to be exclusively symmetrical (Ruch, 1990). However, the upper time limit of four seconds can not be applied to laughter; almost half of them last indeed more than four seconds and the longer lasting episodes of laughter were displayed at funnier jokes than the shorter lasting (Ruch, 1990). 
Thus, the joint contraction of the zygomatic major and the orbicularis oculi muscles forms the common link between smiling and laughter as displays of enjoyment. Moreover, smiles and laughs were shown to represent different degrees of enjoyment rather than being displayed at different stimuli (Ruch, 1990), justifying their coding on one common dimension.

Thus, in light of the above considerations, the hypothesis regarding extraversion and positive affect can be refined by restricting the validity to enjoyment smiles and laughs: Extraverts will show enjoyment displays (smiles and laughter) more often than introverts. Furthermore, extraverts will show the more intense enjoyment displays (i.e., laughter) more frequently than introverts. Smiles based on other than the zygomatic major muscle, not involving the orbicularis oculi or being otherwise classified as false will not differentiate between extraverts and introverts. Alcohol, Enjoyment, and Extraversion

Alcohol dampens excitation in the CNS. Its primary depressant activity is not prevalent in the cortex but in lower centers. The reticular formation is especially affected and consequently its integrating role on the cortex, thalamus, and the hypothalamus is impaired. Depending on the dose the effects of alcohol include hilarity and talkativeness, but also loss of motor coordination, amnesia, and narcosis. According to Pandina (1982), alcohol doses yielding blood alcohol levels up to $0.5 \%$, from $0.5 \%$ o to $1.0 \%$, from $1.0 \%$ o to $1.5 \%$, and higher than $1.5 \%$ o can be regarded as low, medium, high, and very high doses, respectively. Low doses of alcohol may induce talkativeness, euphoria, or complacency and the effects of medium doses include disinhibition, performance decrements in coordination tests, and the beginning of driving impairment (Forth, Henschler \& Rummel, 1975).

Informal as well as formal evidence suggests that consumption of alcohol changes the individuals' affective state, however, the nature of these changes is not entirely clear (McCollam, Burish, Maisto \& Sobell, 1980). High doses of alcohol seem to facilitate negative affect while the effects of intoxication with low and medium doses includes elevation of positive affect; typically 
an increase in scales measuring positive mood states (e.g., elation) is reported (Burish, Maisto \& Shirley, 1982; Connors \& Maisto, 1979; Vuchinich, Tucker \& Sobell, 1979).

Whereas the mood elevating effects of low and medium doses of alcohol are well documented, there is no evidence so far for the widespread belief (e.g., Morreall, 1983) that ethanol intoxication lowers the threshold of amusement. Cheerful mood has been shown to represent a state of lowered threshold for facial responses to humor (Ruch, 1990). Given that alcohol is able to elevate the level of positive mood, one could expect that low and medium doses of alcohol would facilitate the elicitation of smiling and laughter in response to humor. However, the studies carried out so far were not able to demonstrate a main effect of alcohol on facial and verbal indicators of amusement (Vuchinich et al., 1979; Weaver, Masland, Kharazmi \& Zillmann, 1985). Several factors may account for this. First, no separation of types of smiling and laughter has been undertaken in these studies. Alcohol might also affect the display of smiles reflecting negative emotions (e.g., contempt) or facilitate other types of smiling. The presence of other types of smiles/laughter in addition to enjoyment displays might level existing effects. Secondly, besides changing the affective state, higher doses of alcohol might also impair the understanding of humor. Thus, a facilitation of positive affects might be restricted to blunt humor and does not occur for subtle humor. Weaver et al. (1985) report of such an interaction between intoxication and type of humor, however, it was found only for rated funniness but not for facial expression. Third, most importantly, the differential effects of alcohol were not taken into account. Like for other psychotropic drugs, a response variability exists also for alcohol (Janke, 1983). Differential effects might average out, or at least suppress main effects by enlarging the within group variance. These effects can be studied by investigating the interactions between alcohol consumption and the relevant personality traits.

The role of extraversion in accounting for part of the variability of responses to alcohol has been demonstrated quite early (McDougall, 1929). The drug postulate advanced by Eysenck 
$(1957,1983)$ considers extraversion to be a major moderator of the effects of alcohol. Based on the inhibition theory of extraversion it is assumed that:

depressant drugs increase cortical inhibition, decrease cortical excitation and thereby produce extraverted behaviour patterns. Stimulant drugs decrease cortical inhibition, increase cortical excitation and thereby produce introverted behaviour patterns. (p. 229)

Thus, depressant drugs and extraversion broadly have comparable effects on behavior. However, interactive effects are expected as well, based on the postulate that extraverts reach the so-called sedation threshold sooner than introverts. The inhibition theory considers extraverts to be near to the maximal point of inhibition, and depressant drugs can not shift the behavior much more in that direction.

Alcohol often served as a means to illustrate the drug postulate. "Alcohol ... certainly makes many introverts much livelier, more garrulous, and generally more extraverted than normal" (Eysenck \& Eysenck, 1985; p. 194). However, only few of the studies testing the predictions regarding the interaction between extraversion and sedative drugs used alcohol; mainly barbiturates were used (see, for example, Janke, Debus \& Longo, 1979). Those experiments, however, were carried out mainly on performance variables and rarely so in the domain of positive emotion. There is no published study known on the interactive effect of extraversion and alcohol on humorinduced positive affect.

In the present study the effects of alcohol on enjoyment are expected to be different for introverts and extraverts. If frequency and intensity of positive affect go along with extraversion, and depressant drugs make introverts more extraverted, then introverts will display more positive affect under uptake of small and medium doses of alcohol than in a sober condition. Since extraverts are expected to have a lower sedation threshold, one can hypothesize that with increasing doses of alcohol the amount of positive affect decreases for extraverts. Thus, the reduction of positive affect induced by higher doses of alcohol in normals might appear for 
extraverts at comparably lower doses than for ambiverts (which in turn have a lower threshold than introverts). For extraverts it is therefore expected that they will show less positive affect under uptake of a medium alcohol dose than without alcohol.

Thus, the present experiment examines the role of extraversion and consumption of low and medium doses of alcohol on facial and verbal indicators of humor-induced positive affect. Higher doses will not be applied because they would not facilitate positive affect (Forth et al., 1975). For reasons specified elsewhere (McGhee, 1979; Ruch, 1990, 1993), the term "exhilaration" rather than "amusement" or "mirth" will be used to denote the nature of the elicited emotion. According to its Latin root (hilaris = cheerful) the term "exhilaration" is understood as denoting either the process of making cheerful or the temporary rise in cheerful state. Furthermore, as outlined above, it will be examined whether the separation of enjoyment and non-enjoyment displays plays a critical role in verifying the hypothesis that extraversion is a predictor of the frequency and intensity of smiling and laughter.

\section{METHOD}

\section{Subjects}

Female non-psychology students were recruited by advertisements on campus and were paid DM 20 for their participation in this experiment. They were screened according to several criteria, such as medication, current health status, history of alcohol problems, pregnancy, German as a native language, and quality of vision. Subjects were informed upon arrival that the study was designed to test the effects of alcohol intake on finger dexterity and that the whole experiment would be videotaped. Subjects were instructed to avoid eating during the three hours prior to participating in the experiment. When debriefed, they were informed about the real purpose of the study and that the camera served to film their facial responses to the humorous slides rather than their dexterity. All subjects gave consent to have their tapes analyzed. The videotapes of two subjects were partly unscoreable, leaving 61 subjects. Their age ranged from 19 to 30 years, with a mean of 22.9 years and a standard deviation of 3.0 years. 


\section{Materials}

Eysenck Personality Questionnaire-Revised (EPQ-R; Eysenck, Eysenck \& Barrett, 1985). The German version of the EPQ-R is a 102 item questionnaire answered in a yes/no format. It contains four scales: Psychoticism (P: 32 items), Extraversion (E: 23 items), Neuroticism (N: 25 items), and Lie or Social Desirability (L: 22 items). The EPQ-R was administered to the subjects one week before the main experiment.

Eigenschaftswörterliste-Kurzform (EWL-K; Janke \& Debus, 1978). The EWL-K is an adjective list aimed at providing a comprehensive assessment of mood states. The EWL-K contains a list of 123 mood-descriptive adjectives which are answered in a yes/no-format, and provides scores for 14 mood dimensions. These dimensions can be combined to form six domains of mood, and further combined to form the global dimensions of positive and negative affectivity. For the present study two scales were of special importance: positive affectivity and extraversion/introversion (one of the 6 domains).

Aufmerksamkeits-Belastungstest d2 (d2-concentration-test, Brickenkamp, 1978). The $\mathrm{d} 2$ measures the ability to discriminate visually among 16 different elements quickly and correctly. The 16 elements results from combinations of $p$ s or $d$ s with one, two, three, or four commas arranged on the top and/or the bottom of the letter. The subject's task is to mark all $d$ s with two commas on a page containing 14 rows with 47 symbols each. The $\mathrm{d} 2$ was used to assess the capacity for concentration and the number of correctly identified elements (GZ-F) served as a criterion for determining the degree of alcohol-induced impairment of cognitive functions. Both the $\mathrm{d} 2$ and the EWL-K were administered to subjects twice; at the beginning of the experiment and immediately before the period of induction of exhilaration. This allowed for measuring the effects of alcohol on mood state and concentration ability.

\section{Procedure}

Overview. The experiment lasted 90 minutes. Subjects were tested individually by a female experimenter. They first completed the EWL-K and the d2-test. They then received under double 
blind conditions a beverage which they consumed within five minutes. The doses applied can be expected to be effective after 25 to 35 minutes (Forth et al., 1975; Ideström \& Cadenius, 1968; McCollam et al., 1980; Sidell \& Pless, 1971). During this period the subjects completed several dexterity tests and answered the EWL-K and the $\mathrm{d} 2$ again. Subsequently--30 minutes after consumption of the beverage--they were shown slides with jokes and cartoons. Thereafter, they worked on the dexterity tests again. All subjects were offered coffee upon completion of the experiment and subjects in the experimental groups were given special attention while the effects of the alcohol wore off.

Consumption of alcoholic beverage. The beverage consumed by subjects of the control group contained a mixture of peppermint water and grapefruit juice. The low and high alcohol beverage contained this same mixture, plus $0.22 \mathrm{~g}$ or $0.39 \mathrm{~g}$ alcohol per $\mathrm{kg}$ body weight, respectively. A $90 \%$ ethanol was used and was mixed with peppermint water on a 1:1 basis and filled up to 0.21 with grapefruit juice. These manipulations were expected to lead to blood alcohol levels of $0.4 \%$ and $0.7 \%$ (Forth et al., 1975). For the sake of clarity, the two experimental groups are referred to as low and high alcohol groups in the present study, although these levels of blood alcohol should be regarded as low and medium doses. Neither the subject nor the experimenter were aware of the content of the two beverage drinks which were offered to the subjects after they completed the initial tests. The choice of beverage determined under which of the three experimental conditions the subjects took part.

Induction of exhilaration. The exhilaration-induction procedure lasted for 20 minutes. Subjects were presented 35 black and white slides of jokes and cartoons at an interval of 25 seconds while sitting in a comfortable chair in a slightly darkened part of the room. The first five humor stimuli were for "warming up" and were not used in any analyses. The remaining 30 represented equal numbers of the three categories of incongruity-resolution humor, nonsense humor, and sexual humor (Ruch, 1992). The jokes/cartoons of the three categories were brought into a random order, and were presented to subjects in one of two permutations. 
Verbal responses. The subjects were instructed to rate all 35 stimuli according to degree of funniness and aversiveness on two seven-point Likert scales and to indicate in a yes/no-format whether or not they found the humor stimulus tasteless. These scales were selected as markers for the orthogonal humor response dimensions of positive and negative affect which are typically obtained in factor analyses of response scales (Ruch \& Rath, in press). The former scale represents the intensity of positive responses to humor and has been shown to correlate very high with judged degree of felt exhilaration (Ruch \& Rath, in press). The latter two scales cover the negative responses induced by humor.

Facial measurement. Measurements were made from color videotapes, which provided a close-up, head-on view of the subject's face. The measurements were based on the Facial Action Coding System (FACS; Ekman \& Friesen, 1978). The FACS is an anatomically based, comprehensive, objective technique for measuring all observable facial movement. It distinguishes 44 action units (AUs). These are the minimal units that are anatomically separate and visually distinguishable. Facial coding usually requires slowed-motion inspection of recorded behavior. FACS also allows for measurement of the timing of a facial movement, its symmetry and intensity, and its degree of irregularity of onset, apex or offset.

The 1830 potential responses in the present experiment were coded for frequency, intensity, symmetry/asymmetry and duration of five action units by two coders independently from each other. In case of disagreement, they watched the respective responses together until agreement was achieved. Their degree of agreement--estimated on the basis of other material--was $93.1 \%$.

The intensity and symmetry/asymmetry of the action units were coded during the apex of their occurrence. Three intensity grades (slight, moderate, extreme) were distinguished according to the criteria given in the FACS manual. For statistical treatment of the data the three grades were assigned values from 1 to 3 , and the absence of any facial action was coded as 0 . The asymmetry was determined by judging whether the appearance changes of the AU were stronger in the left or 
right half of the face. The duration was defined as the time interval from the first noticeable appearance of the characteristic changes of an AU to their complete disappearance.

On the basis of the coded material several displays were distinguished. The enjoyment smile was defined by the presence of two action units, the AU12 and AU6, with no further facial actions present. AU12 ("lip corner puller") refers to the contraction of the zygomatic major. AU6 ("cheek raiser") describes the actions of the outer part of the orbicularis oculi muscle. The AU12 should be symmetrical and last longer than $2 / 3$ of a second. The enjoyment laugh was coded when laughter vocalization or audible respiration changes occurred together with a joint symmetric action of AU12 and AU6 which lasted longer than 2/3 seconds. Episodes of a single audible forced expiration accompanied by an AU12 and AU6 formed the lower end of the intensity spectrum of events coded as laughter. However, a fully developed laughter pattern was generally observed which typically consisted of an initial forced exhalation, followed by a more or less sustained sequence of repeated expirations of high frequency and low amplitude, which may or may not be phonated as "ha-ha-ha." Enjoyment smiles and enjoyment laughs were combined to form the category of enjoyment displays.

The category of non-enjoyment displays consisted of three subgroups: 1) AU12-smiles which were not accompanied by an AU6, or asymmetrical and of short duration. 2) smiles involving AU13.3) smiles involving AU14. AU13 ("cheek puffer") refers to the contraction of the caninus muscle which pulls the lip corner up sharply and puffs up cheeks. AU14 ("dimpler") describes the dimplelike wrinkles at lips corners produced by the contraction of the buccinator muscle. Both types of smiles were observed to appear alone or together with AU12.

The frequency of these displays was determined by counting how often they occurred in response to the 30 slides. Two indices for intensity of enjoyment displays were computed. Both of them were independent of the frequency and were based on an ordering of the responses on a dimension including the levels "no response" (=0), three intensity levels of "enjoyment smiles" $(1,2$, and 3, representing the three intensity grades of the AU12 in AU12+AU6 displays) and 
"laughter" (=4). The maximal intensity was represented by the highest level of facial enjoyment coded for a subject. This five-point index ranged from 0 to 4 . A score for the average intensity was derived as well by adding the five-point intensity index across all slides and dividing the sum by the frequency of enjoyment displays. For the ANOVAs composite scores were computed which combined both frequency and intensity of facial displays. The intensity of enjoyment displays was derived by adding the five-point intensity index across all enjoyment displays. A similar composite score was obtained for intensity of non-enjoyment displays; this score was much lower since the AU13 or AU14 in the individual responses did not exceed the medium intensity level.

\section{RESULTS}

The Extraversion in the present sample $(M=14.9, S D=4.5, \alpha=0.84)$ yields results comparable to the German norms for females of this age range. Subjects were classified into introverts ( $n=20$, score range from 5 to 12 points on the E scale), ambiverts ( $n=20$, from 13 to 17 ) and extraverts $(n=21$, from 18 to 22$)$ which were distributed randomly $\left(\chi^{2}[4]=1.632, p=\right.$ 0.803 ) over the control group, low, and high alcohol groups with the number of subjects in the resulting nine groups ranging from 6 to 9 .

For mood and concentrative ability, 3 x 3 ANOVAs with alcohol consumption as treatment variable (no ethanol, low dose, high dose) and extraversion as classification variable (introverts, ambiverts, extraverts) were computed for difference scores (second minus first administration) in elation, state extraversion/introversion (EWL domain scale), and the d2-test total score (GZ-F). For the facial data, a 3 × 3 × 2 ANOVA (alcohol consumption x extraversion $x$ type of display) with intensity of enjoyment and non-enjoyment displays as a repeated measurement factor was computed. For the verbal data, 3 x 3 ANOVAs (alcohol consumption x extraversion) with funniness, aversiveness, and tastelessness as dependent variables were performed. Effects of Extraversion and Alcohol on Mood State and Concentrative Ability. 
The effect of alcohol on change in the extraversion/introversion-scale of the EWL just failed to reach significance (see Table 1). Nevertheless, post hoc comparisons revealed the expected alcohol-induced change into a more extraverted state for the high dose group, whereas subjects of the control group appeared more introverted at the second administration of the EWL than at the first administration. This difference was significant ${ }^{1}$.

Insert Table 1 about here

Elation increased between the low and high alcohol dose (see Table 1). However, the analysis of the alcohol-extraversion interaction $(\mathrm{F}[4,52]=3.905, p=0.008)$ revealed that this was true only for introverts $(\mathrm{F}[1,52]=9.788, p=0.003)$ and extraverts $(\mathrm{F}[1,52]=7.323, p=$ 0.009); for ambiverts elation tended to decrease $(\mathrm{F}[1,52]=3.457, p=0.069)$.

The administration of alcohol impaired cognitive ability (see Table 1). Although all groups yielded higher scores on the second administration of the test of concentrative ability, the increase was lower for the low and high alcohol group as compared to the controls.

Effects of Extraversion and Alcohol on Responses to Humor.

First, the effects involving the separation of type of display were evaluated. The trivial main effect on the repeated measurement factor confirmed that the stimuli evoked more enjoyment displays than non-enjoyment displays, $\mathrm{F}[1,52]=42.184, p<0.0001$. There was no interaction between alcohol and type of display, $\mathrm{F}[2,52]=0.112, p=0.895$.

Extraversion and enjoyment and non-enjoyment displays. As expected, there was an interaction between extraversion and type of display, $\mathrm{F}[2,52]=3.860, p=0.027$. The nature of this interaction is displayed in Figure 1.

\footnotetext{
${ }^{1} \mathrm{An}$ analysis of the non-significant interaction between alcohol and extraversion $(\mathrm{F}[4,52]=1.975$, $p=0.129$ ) showed that for ambiverts the increase in extraverted state occurred between low and no alcohol condition $(\mathrm{F}[1,52]=4.340, p=0.042)$ whereas for introverts the increase could be observed between the low and high dose conditions $(\mathrm{F}[1,52]=5.669, p=0.021)$.
} 
Insert Figure 1 about here

Figure 1 shows that the intensity of the non-enjoyment display does not increase as a function of the subjects' degree of extraversion. For the enjoyment displays, however, the extraverts show more intense displays than the introverts $(\mathrm{F}[1,52]=8.785, p=0.005)$ and the ambiverts $(F[1,52]=4.526, p=0.038)$. Because of the more frequent occurrence of enjoyment displays, the main effect for extraversion $(\mathrm{F}[2,52]=5.007, p=0.010)$ becomes significant. Nevertheless, Figure 1 confirms that it is not justified to lump enjoyment and non-enjoyment smiles together. For the verbal data no separation of types of response was undertaken; the main effect of extraversion failed to reach significance (funniness: $\mathrm{F}[2,52]=2.682, p=0.078$ ) although extraverts again tended to find the stimuli more funny than the introverts $(p=0.067)$ and the ambiverts $(p=0.041)$.

Extraversion, alcohol, and enjoyment of humor. There is an interaction between extraversion and alcohol for both the facial $(\mathrm{F}[4,52]=3.611, p=0.011)$ and the verbal $(\mathrm{F}[4,52]$ $=2.628, p=0.045)$ indicator of exhilaration. For the facial data, this interaction seems to be moderated by the type of facial display as indicated by the triple interaction between extraversion, alcohol, and type of enjoyment $(\mathrm{F}[4,52]=2.513, p=0.053)$, which just fails to be significant. Separate 3 x 3 ANOVAs for enjoyment and non-enjoyment displays confirmed that the interaction between alcohol and extraversion is far from being significant for the non-enjoyment displays ( $F$ $[4,52]=1.086, p=0.373)$ but significant for the enjoyment displays $(\mathrm{F}[4,52]=3.219, p=$ 0.019). The nature of this interaction is presented in Figures $2 \mathrm{a}$ and $2 \mathrm{~b}$ for the intensity of the enjoyment display and the verbal data, respectively.

Insert Figures $2 \mathrm{a}$ and $2 \mathrm{~b}$ about here 
Figure 2 shows that for introverts there is no difference in the magnitude of facial or verbal exhilaration between the control group and the average of the two alcohol groups $(\mathrm{F}[1,52]=$ $0.161, p=0.690$; funniness: $\mathrm{F}[1,52]=0.531, p=0.469)$. However, extraverts in the high alcohol group do show significantly less facial enjoyment than extraverts in the control group ( $\mathrm{F}$ $[1,52]=7.098, p=0.010)$ and they also rate them less funny $(\mathrm{F}[1,52]=8.515, p=0.005)$. The reduction of exhilaration in extraverts can already be observed for the low alcohol group (enjoyment display: $\mathrm{F}[1,52]=5.447, p=0.023$; funniness: $\mathrm{F}[1,52]=3.107, p=0.084$ ).

Ambiverts were expected to display the same response pattern as the introverts. Post hoc tests revealed that ambiverts showed indeed more facial enjoyment in the low alcohol group than in the control group (enjoyment display: $\mathrm{F}[1,52]=4.662, p=0.036$; funniness: $\mathrm{F}[1,52]=2.444$, $p=0.124$ ); there is no difference among the two alcohol groups (enjoyment display: $\mathrm{F}[1,52]=$ $0.828, p=0.367$; funniness: $\mathrm{F}[1,52]=1.908, p=0.173)$. Thus, for ambiverts the facilitative effect of low doses of alcohol can be found in the low alcohol group which was expected to occur for introverts. Thus, there were interactive effects of alcohol on humor-induced exhilaration; this leads to the question whether these effects are mediated by changes in positive affectivity.

Can positive affectivity be seen as a variable mediating the effects of alcohol on humor-induced exhilaration? Individual differences in the EWL-scale positive affectivity correlated with facial $(R=0.326, p=0.011$; control group: $R=0.52, p=0.023)$ and verbal $(R=$ $0.276, p=0.033$ ) enjoyment. Also the rank-order of the cell means of positive affectivity and the intensity of enjoyment displays $(R=0.783, p=0.027)$ were correlated. No such correlations appeared for positive affectivity of the first administration. Subsequently, a 3 x 3 ANCOVA (treatment $\mathrm{x}$ extraversion) with positive affectivity as covariate was performed for intensity of enjoyment displays and rated funniness as dependent variables. The main effect for extraversion was not significant (enjoyment display: $\mathrm{F}[2,43]=0.369, p=0.694$; funniness: $\mathrm{F}[2,43]=1.556$, $p=0.223$ ) and so was the extraversion alcohol interaction (enjoyment display: $\mathrm{F}[4,43]=0.165$, $p=0.955$; funniness: $\mathrm{F}[4,43]=0.824, p=0.517)$. Thus, once the effects due to different 
degrees of positive affectivity were removed, none of the effects of alcohol on humor-induced exhilaration remained. Neither positive affectivity at the first administration of the EWL nor the difference scores in positive affectivity were nearly as effective in reducing the explained variance.

Changes in the level of extraverted state can not be seen as mediators. Whereas individual differences in EWL-extraversion/introversion at the second administration did correlate with facial $(R=0.27, p=0.037$; control group: $R=0.536, p=0.019)$ and verbal $(R=0.299, p=0.020)$ indicators of exhilaration, the pattern of means did not match and it turned out that extraverted state can not account for the effects in the ANCOVA.

Alcohol and humor-induced positive and negative affect. There was no main effect of alcohol on humor-induced exhilaration (enjoyment display: $\mathrm{F}[2,52]=0.316, p=0.731$; funniness: $\mathrm{F}[2,52]=0.528, p=0.593)$. However, there were main effects on intensity of the non-enjoyment displays and on the negative evaluation of the stimuli (see Table 2).

Insert Table 2 about here

Pairwise comparisons showed that there were more non-enjoyment displays in the low alcohol group than in the control group and in the high alcohol group whereas the latter two groups did not differ from each other. Inspection of the means showed that this effect was obtained for both forms of non-enjoyment displays: the smiles based on AU13 or AU14, and the AU12-smiles lacking the markers for enjoyment (AU6, symmetry, duration), although it was more pronounced so in the former. Similarly, the stimuli were judged more negatively in the low alcohol group than in the control group and in the high alcohol group (for tasteless: $p=0.053$ ) whereas the latter two groups did not differ from each other.

Thus, the same effects emerged for aversiveness and intensity of the non-enjoyment displays. In fact, there was a significant positive correlation between degree of rated negative affect and intensity of smiles based on AU13 or AU14 (aversive: $R=0.341, p=0.008$; tasteless: 
$R=0.343, p=0.008)$ but not with the AU12 without participation of the AU6 $(R=-0.058, p=$ 0.65 ; tasteless: $R=0.107, p=0.407)$. The latter correlated highly with rated funniness $(R=$ $0.561, p<0.001)$ but also the former did correlate positively $(R=0.281, \mathrm{p}=0.030)$. Extraversion and Frequency and Intensity of Positive Affect.

The hypotheses regarding the relationship between extraversion and facial and verbal indicators of positive affect were investigated in more detail. Rank order correlations between the Extraversion scale of the EPQ and the frequency and intensity of several action units are computed for the control group (i.e., sober subjects) and are presented in Table 3. For purposes of comparison the coefficients obtained for the total sample of 61 subjects are provided as well.

Insert Table 3 about here

Table 3 shows that extraversion does correlate with both the frequency and the intensity of facial indicators of exhilaration in the control group as well as in the total sample. As regards the frequency of displays, extraverts show all forms of Duchenne-displays (AU12+AU6) more often than introverts, regardless of whether they were or were not accompanied by vocalizations (i.e., were smiles or laughs). As regards the intensity of displays, extraversion correlates with both the maximal intensity displayed and the average intensity. Both indices are logically but not empirically independent of the frequency $(R \mathrm{~s}=0.73$ and 0.581 , for the subjects showing enjoyment displays); they are also highly intercorrelated $(R=0.845$, all $p \mathrm{~s}<0.0001)$. Not surprisingly, the coefficient obtained for the composite score of facial enjoyment (covering both frequency and intensity) is highest in both the control group as well as the total sample. As regards the verbal indicators of exhilaration, there appears to be a weaker relationship. The correlation between extraversion and rated funniness is lower and even fails to be significant in the total sample.

The validity of the separation of types of displays is also evident in the comparison of the size of the coefficients obtained for them. The correlation between extraversion and the number of 
all AU12 displayed by a person (i.e., regardless of whether they were or were not accompanied by an AU6) is of medium size and significant. This correlation, however, is low and insignificant when computed for the smiles not involving the eye region, and much higher and significantly so, when computed for responses including the characteristic changes due to contraction of the orbicularis oculi. In the control group, extraverts also tend to display smiles based on AU13 or AU14 more often than introverts.

Finally, it was investigated whether introverts are agelasts (i.e., non-laughers). Of the 61 subjects, 33 subjects did not laugh at all and 9 subjects did not even display one single enjoyment smile (i.e., a joint action of AU12 and AU6 without vocalization). It turns out that introverts are overrepresented among the agelasts; 14 out of 20 (70\%) introverts did not laugh, but there were only 8 out of $21(38.1 \%)$ extraverts who did not laugh $\left(\chi^{2}[1]=4.193, p=0.041\right)$. Similarly, all extraverts displayed at least one single enjoyment smile, but $5(25 \%)$ of the introverts failed to do so $\left(\chi^{2}[1]=5.979, p=0.014\right)$. Thus, the difference between extraverts and introverts relates not only to the question how often a certain facial expression is displayed or how intense it is but already to whether or not it is displayed at all.

\section{DISCUSSION}

The present study shows that extraverts tend to exhibit facial signs of positive affect more frequently than introverts. This tendency is restricted to enjoyment displays and cannot be found for other sorts of smiles/laughter. Thus, the verification of this hypothesis is contingent on the separation of smiles according to their morphology. Only responses including a joint action of the zygomatic major muscle and the orbicularis oculi muscle, which are symmetric and last longer than 2/3 seconds discriminate among extraverts and introverts. Smiles based on a principal muscle other than the zygomatic major, or false smiles (not including action of the orbicularis oculi; asymmetric smiles, or smiles of short duration) do not vary as a function of the degree of extraversion. Thus, it is possible that the study by Shimizu et al. (1982) did fail to find a 
difference between extraverts and introverts because of the lack of separation of enjoyment displays and non-enjoyment smiles and laughs. Similarly, a recent study by Jäncke (1993) did not find a significant difference between extraverts and introverts (as measured by the E scale of the EPQ-R) and zygomatic major activity as induced by pictures of positive valence.

The results show that extraversion is associated with both, the frequency and the intensity of positive affect as displayed in the face. Hence, the hypotheses put forward by Eysenck and Eysenck (1985) is supported also outside the domain of self-reported mood. Extraverts did show all types of enjoyment displays more frequently than introverts. Furthermore, the average and the maximal intensity of the enjoyment display increased with increasing degrees of extraversion. The coefficients appear to be lower for intensity than for frequency, however, this might be due to the lower reliability of the former. The maximal intensity score is based on one response only which inevitably lowers the reliability. Besides this, its five-point format does not provide much discrimination among subjects. The reliability of the average intensity score is lowered by the fact that the base rates of enjoyment-laughs and enjoyment-smiles were low in the present study (as they are in other laboratory studies as well). Several subjects did not show laughter at all and some subjects showed only a few smiles. A higher rate of enjoyment displays would allow for a more reliable estimation of its habitual intensity. This could be achieved by using more and/or funnier stimuli or by providing situational conditions which facilitate the release of exhilaration in an experimental setting. Nevertheless, due to the fact that the intensity indices were logically independent of the frequency, the results of the present study can be regarded as supporting the intensity hypothesis.

The results also show that extraverts do indeed "like to laugh" (Eysenck \& Eysenck, 1975). Since laughing occurs at higher levels of positive affect and smiling is typical of lower levels (Ruch, 1990), this result for laughter falls in line with the ones obtained for the intensity hypothesis. In fact, for non-intoxicated subjects (i.e., in the control group) the correlation between extraversion and laughter was the highest obtained for all individual behavioral indicators of 
positive affect. The relationship between extraversion and laughter is not restricted to humorinduced laughter, or to laughter induced in the laboratory. Ruch and Deckers (in press) found that extraversion and a questionnaire assessing the propensity to laugh and smile in a variety of everyday situations correlated 0.52 and 0.36 in German and American samples, respectively.

The low size of the correlation between extraversion and judged funniness of the jokes and cartoons deserves separate attention. The results seem to suggest that extraversion does affect facial displays of emotion but less so the affective experience. There might be two reasons for this fact. Firstly, all ratings were summed up including those which were accompanied by no facial display or even a false smile. It might be that their exclusion would have yielded different results. Such an analysis was not undertaken, however, since this might have artificially duplicated the results found for the facial data. Secondly, there is no reason to assume that extraverts smile and laugh more than introverts when they do not appreciate the stimuli presented. In fact, extraverts can not even be expected to smile if they do not like the material. Thus, the difference between extraverts and introverts with respect to the amount of laughing can be expected to appear only when other factors, like humor preferences, are kept constant. The use of different types of humor reduced the variance with respect to differential humor preference. Nevertheless, there are still tremendous individual differences with respect to the degree of appreciation of canned humor remaining as seen in the variance of the funniness scores. This variance is not considered to reflect only state variance related to individual differences in the easiness of inducing positive affect but seems to contain variance related to humor preference. A more stringent test of the hypothesis would therefore include an elimination of this variance and the analysis of the variance relating only to the threshold of induced positive affect. For example, subjects could be divided into those who appreciate the material and those who do so less. For the group of Ss who do not appreciate the humor very much no difference between extraverts and introverts with respect to the amount of smiling/laughter can be expected. However, among those who appreciate the humor the extraverts will show enjoyment displays more frequently than introverts. 
As expected, the effects of alcohol on mood or humor-induced exhilaration were not uniform. The consumption of a moderate dose of alcohol did raise the subjects' level of stateextraversion. Also, state-extraversion did correlate with the facial and verbal indicators of exhilaration albeit lower so than trait-extraversion. However, the consumption of a moderate dose of alcohol did not generally facilitate the induction of exhilaration; neither facial displays nor verbal judgements of funniness increased as a function of alcohol. This replicates the negative results found in earlier studies on the effects of alcohol on humor-induced laughter (Vuchinich et al., 1979; Weaver et al., 1985).

Alcohol did raise elation and impaired the capacity to concentrate. However, this apparently did not affect responses to humor as inferred from the different pattern of means. A low dose of alcohol did raise the negative evaluations of the humor stimuli and facilitated the release of nonenjoyment displays. Whereas the meaning of the AU13 is still unknown, the joint asymmetric action of the AU14 and AU12 is the facial expression of contempt and hence its association with higher aversiveness ratings is not surprising. The sole action of the AU12 was shown to accompany very low levels of enjoyment of humor (Ruch, 1990). Thus, a low dose of alcohol seem to create a transition state which is characterized by the facilitation of emotionality in the range of negative to mildly positive affect.

The effects of alcohol on humor-induced positive affect are moderated by extraversion. The interactive effects on the verbal and facial indicators of exhilaration are highly comparable. However, the predictions based on the drug postulate did receive only partial support. As predicted, extraverts showed less verbal and facial exhilaration in the high alcohol condition than in the control group. The results indicate that even a low dose reduces the enjoyment of extraverts. However, contrary to the expectations, the introverts' verbal and facial behavior was not affected by alcohol consumption. It was the group of ambiverts which partly displayed the behavioral pattern expected to occur for introverts. 
One reason for the failure to find facilitative effects for introverts could be that the doses used were too low to be effective in introverts; doses higher than $0.7 \%$ o would have been needed. This argument is supported by the findings that introverts in the high alcohol group did describe themselves as being more extraverted and in a more positive mood than in the low alcohol group; the behavioral pattern, however, has not yet altered accordingly. Another argument is that the base rate of enjoyment displays among introverts $(M=2.8)$ is too low to reliably reflect the facilitative effects of alcohol. Even if alcohol has facilitating effects on laughter; the threshold for the release of laughter might still be too high for introverts to be exceeded often enough. The application of the suggestions made above might help to rule out this interpretation. So far, the results of the present study seem to be in line with McDougall's (1929) conclusion that the introvert is much more resistant to alcohol than the extravert.

The results are compatible with the view that positive mood states serve as intervening variables accounting for the effects of alcohol and extraversion on humor-induced exhilaration. Individual differences in positive affectivity predicted the differences in degree of exhilaration within and among experimental groups. Once the level of positive mood was kept constant, the effects of extraversion and alcohol diminished almost completely. Positive affectivity seems to represent a state of lowered threshold for the induction of exhilaration just as cheerful mood-which is one segment of positive affectivity--does (Ruch, 1990). Extraversion and alcohol created different levels of positive mood, which, in turn, represented different degrees of preparedness for the release of smiling and laughter. The results also allow for the speculation that the extraverts' higher frequency and intensity of enjoyment displays might be due to the fact that positive affectivity is more strongly prevalent among extraverts than among introverts.

The results of the present experiment underscore the necessity of including extraversion in future studies of the effects of alcohol on humor-induced positive affect. Likewise, the separation of enjoyment displays from other sorts of smiles/laughter appears necessary for the verification of hypotheses. 
Extraversion, alcohol, and enjoyment - 25 -

\section{Author Notes}

This manuscript is based on data of the habilitation thesis submitted to the School of Mathematical and Natural Sciences at the University of Düsseldorf. The preparation of this manuscript was facilitated by a Heisenberg grant from the German Research Council (DFG) to the author. The manuscript was written during my stay at the Department of Psychology, University of Bielefeld. Correspondence and requests for reprints should be addressed to Willibald Ruch, Department of Physiological Psychology, Heinrich-Heine University of Düsseldorf, Universitätsstraße 1, 4000 Düsseldorf, Germany. 
Extraversion, alcohol, and enjoyment - 26 -

\section{REFERENCES}

Brickenkamp, R. (1978). Test d2. Aufmerksamkeits-Belastungs-Test. Göttingen: Hogrefe.

Burish, T. G., Maisto, S. A. \& Shirley, M. C. (1982). Effect of alcohol and stress on emotion and physiological arousal. Motivation and Emotion, 6, 149-159.

Connors, G. J. \& Maisto, S. A. (1979). Effects of alcohol, instructions, and consumption rate on affect and physiological sensations. Psychopharmacology, 62, 261-266.

Costa, P. T. \& McCrae, R. R. (1980). Influence of extraversion and neuroticism on subjective wellbeing: Happy and unhappy people. Journal of Personality and Social Psychology, 38, 668678.

Ekman, P. (1985). Telling lies. New York: Berkley Books.

Ekman, P., Davidson, R. J. \& Friesen, W. V. (1990). The Duchenne smile: Emotional expression and brain physiology, II. Journal of Personality and Social Psychology, 58, 342-353.

Ekman, P. \& Friesen, W. V. (1978). Facial action coding system (FACS). Palo Alto, CA: Consulting Psychologists Press.

Ekman, P. \& Friesen, W. V. (1982). Felt, false, and miserable smiles. Journal of Nonverbal Behavior, 6, 238-252.

Eysenck, H. J. (1957). Drugs and personality, I. Theory and methodology. Journal of Mental Science, 103, 119-131.

Eysenck, H. J. (1983). Drugs as research tools in psychology: Experiments with drugs in personality research. Neuropsychobiology, 10, 29-43.

Eysenck, H. J. \& Eysenck, M. W. (1985). Personality and individual differences: A natural science approach. New York: Plenum Press.

Eysenck, H. J. \& Eysenck, S. B. G. (1975). Manual of the Eysenck Personality Questionnaire. London: Hodder \& Stoughton.

Eysenck, S. B. G., Eysenck, H. J. \& Barrett, P. (1985). A revised version of the psychoticism scale. Personality and Individual Differences, 6, 21-29. 
Forth, W., Henschler, D. \& Rummel, W. (1975). Allgemeine und spezielle Pharmakologie und Toxikologie . Mannheim: Bibliographisches Institut.

Frank, M. G. \& Ekman, P. (1993). Not all smiles are created equal: The differences between enjoyment and nonenjoyment smiles. Humor, 6, 9-26.

Hepburn, L. \& Eysenck, M. W. (1989). Personality, average mood and mood variability. Personality and Individual Differences, 9, 975-983.

Ideström, C. D. \& Cadenius, B. (1968). Time relations of the effects of alcohol compared to placebo. Psychopharmacologia, 13, 189-200.

Janke, W. (1983). Response Variability in Psychotropic Drugs: Overview of the main approaches to differential pharmaco-psychology. In: W. Janke (Eds.), Response variability in psychotropic drugs. London: Pergamon Press.

Janke, W. \& Debus, G. (1978). Die Eigenschaftswörterliste (EWL). Göttingen: Hogrefe.

Janke, W., Debus, G. \& Longo, N. (1979). Differential psychopharmacology of tranquilizing and sedating drugs. In Th. A. Ban et al. (Eds.), Modern problems of pharmacopsychiatry (Vol. 14, pp. 13-98), Basel: Karger.

Jäncke, L. (1993). Different facial EMG-reactions of extraverts and introverts to pictures with positive and negative valence. Personality and Individual Differences, 14, 113-118.

Larsen, R. J. \& Ketelaar, T. (1989). Extraversion, Neuroticism and susceptibility to positive and negative mood induction procedures. Personality and Individual Differences, 10, 12211228.

McCollam, J. B., Burish, T. G., Maisto, S. A. \& Sobell, M. B. (1980). Alcohol's effects on physiological arousal and self-reported affect and sensations. Journal of Abnormal Psychology, 89, 224-233.

McDougall, W. (1929). The chemical theory of temperament applied to introversion and extraversion. Journal of Abnormal and Social Psychology, 24, 293-309.

McGhee, P.E. (1979). Humor: Its origin and development. New York: Freeman. 
Morreall, J.M. (1983). Taking laughter seriously. Albany, NY: State University of New York Press. Pandina, R. J. (1982). Effects of alcohol on psychological processes. In E. Gomberg, H. White \& J. Carpenter (Eds.), Alcohol, science, and society revisited. Ann Arbor: University of Michigan Press.

Ruch, W. (1990). Die Emotion Erheiterung: Ausdrucksformen und Bedingungen . Unpublished Habilitation thesis, University of Düsseldorf, Germany.

Ruch, W. (1992). Assessment of appreciation of humor: Studies with the 3 WD humor test. In C. D. Spielberger \& J. N. Butcher (Eds.), Advances in personality assessment (Vol. 9, pp. 3487). Hillsdale, NJ: Lawrence Erlbaum Associates.

Ruch, W. (1993). Exhilaration and humor (Chapter 42). In: M. Lewis \& J.M. Haviland (Eds.), The Handbook of Emotion. New York, NY: Guilford Publications, 605-616.

Ruch, W. \& Deckers, L. (in press). Do Extraverts 'like to laugh': An analysis of the Situational Humor Response Questionnaire (SHRQ). European Journal of Personality.

Ruch, W. \& Rath, S. (in press). The nature of humor appreciation: Toward an integration of perception of stimulus properties and affective experience. Humor.

Shimizu, A., Kawasaki, T., Azuma, T., Kawasaki, M. \& Nishimura, T. (1982). Polygraphic study on laughing in normals and schizophrenic patients. In M. Namba \& H. Kaiya (Eds.), Psychobiology of schizophrenia (pp. 115-120). Oxford: Pergamon.

Sidell, F. R. \& Pless, J. E. (1971). Ethyl alcohol: Bloodlevels and performance decrements after oral administration to men. Psychopharmacologia, 19, 246-261.

Vuchinich, R. E., Tucker, J. A. \& Sobell, M. B. (1979). Alcohol, expectancy, cognitive labeling, and mirth. Journal of Abnormal Psychology, 88, 641-651.

Weaver, J. B, Masland, J. L., Kharazmi, S. \& Zillmann, D. (1985). Effect of alcoholic intoxication on the appreciation of different types of humor. Journal of Personality and Social Psychology, 49, 781-787. 
Extraversion, alcohol, and enjoyment - 29 -

Table 1. Changes in mood and concentration ability (post-prae) as a function of alcohol consumption

\begin{tabular}{lccccc}
\hline & no alcohol low dose high dose F $[2,52]$ & $p$ \\
\hline extraversion/introversion & $-1.100^{\mathrm{a}}$ & -0.524 & $0.850^{\mathrm{b}}$ & 2.759 & 0.073 \\
elation & -0.100 & $-0.762^{\mathrm{a}}$ & $0.900^{\mathrm{b}}$ & 2.570 & 0.086 \\
GZ-F & $82.150^{\mathrm{b}}$ & $58.238^{\mathrm{a}}$ & $63.150^{\mathrm{a}}$ & 3.551 & 0.036 \\
\hline
\end{tabular}

Note. $a, b$ Means of a row with different superscripts differ at $P$ $<0.05$ by two-tailed t-Tests. 
Extraversion, alcohol, and enjoyment - 30 -

Table 2. Means scores of the effect of alcohol consumption on nonenjoyment displays and negative evaluation of the stimuli

no alcohol low dose high dose $F[2,52] \quad p$

$\begin{array}{lrrrrr}\text { non-enjoyment display } & 2.850^{\mathrm{a}} & 4.571^{\mathrm{b}} & 2.350^{\mathrm{a}} & 5.043 & 0.010 \\ \text { aversive rating } & 12.450^{\mathrm{a}} & 28.905^{\mathrm{b}} & 15.600^{\mathrm{a}} & 5.971 & 0.005 \\ \text { tasteless rating } & 3.280^{\mathrm{a}} & 3.571^{\mathrm{b}} & 3.395 & 4.845 & 0.012\end{array}$

Note. $a, b$ Means of a row with different superscripts differ at $P$ $<0.05$ by two-tailed t-Tests. 
Table 3. Correlations between Extraversion and facial and verbal responses to humor

\begin{tabular}{|c|c|c|c|c|}
\hline & \multicolumn{2}{|c|}{ total sample 1} & \multicolumn{2}{|c|}{ control group ${ }^{2}$} \\
\hline & $R$ & $p$ & $R$ & $p$ \\
\hline \multicolumn{5}{|l|}{ Frequency } \\
\hline AU12+AU6 & 0.404 & 0.002 & 0.553 & 0.016 \\
\hline without vocalization & 0.388 & 0.003 & 0.520 & 0.023 \\
\hline with vocalization & 0.307 & 0.017 & 0.567 & 0.013 \\
\hline \multicolumn{5}{|l|}{ Intensity } \\
\hline maximal intensity & 0.356 & 0.006 & 0.407 & 0.076 \\
\hline average intensity & 0.282 & 0.029 & 0.386 & 0.093 \\
\hline \multicolumn{5}{|c|}{ Global Measures of Exhilaration } \\
\hline rated funniness & 0.229 & 0.076 & 0.458 & 0.046 \\
\hline facial enjoyment & 0.400 & 0.002 & 0.570 & 0.013 \\
\hline
\end{tabular}

Separation of Action Units
all AU12
0.273
0.035
0.474
0.039
AU12 without AU6
$\begin{array}{lll}0.122 & 0.344 & 0.308\end{array}$
0.179
all non-enjoyment displays
0.160
0.215
0.356
0.121
$\mathrm{AU} 13$ and $\mathrm{AU} 14$
$\begin{array}{lll}0.141 & 0.277 & 0.468\end{array}$
0.041

Note. $1 n=61 ; 2 n=20$. 
Extraversion, alcohol, and enjoyment - 32 -

\section{Figure Captions}

Figure 1. Facial Responsiveness as a function of type of display and extraversion.

Figure 2. Intensity of enjoyment display (a) and judged funniness (b) as a function of alcohol consumption and extraversion. 


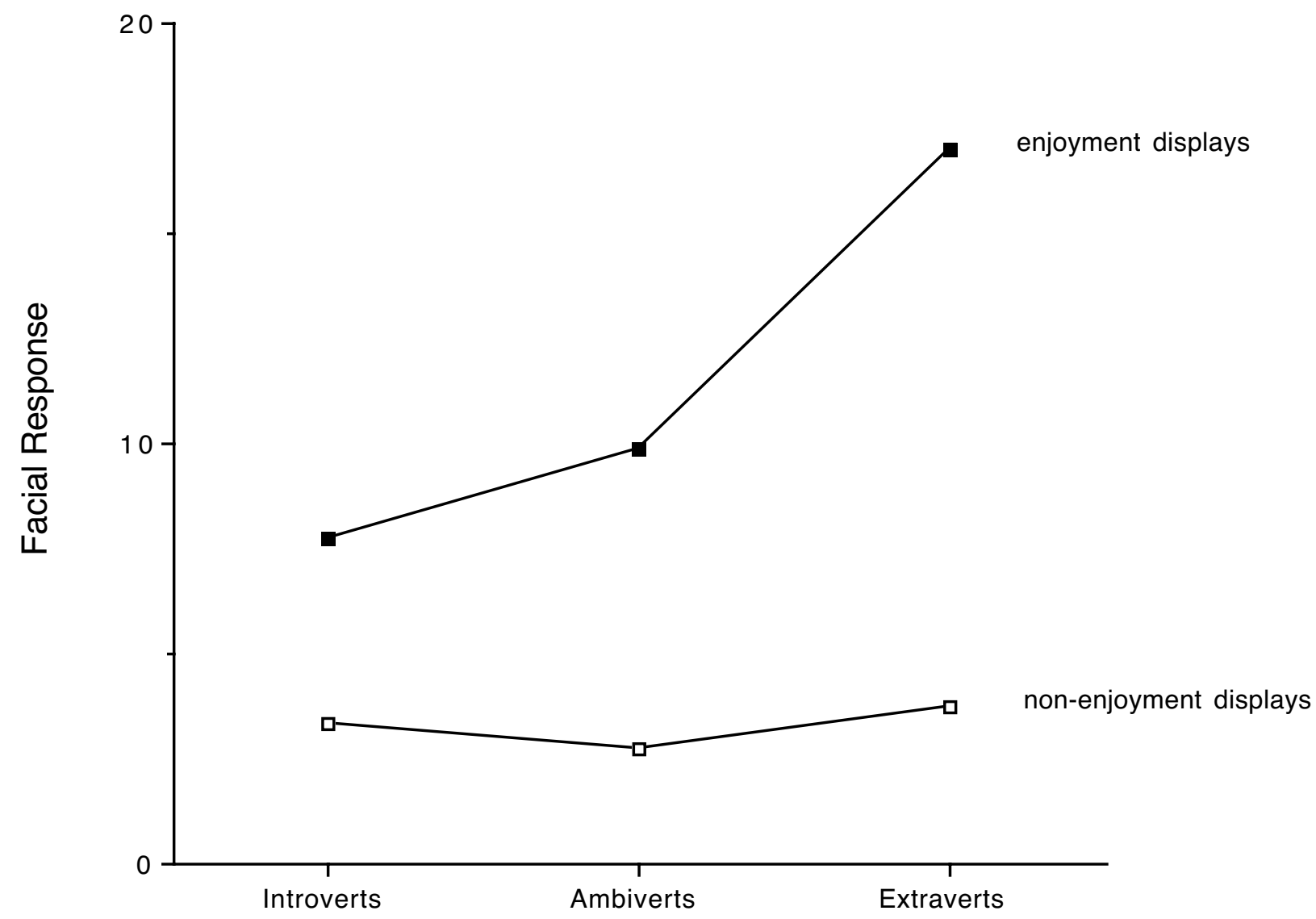

Extraversion 


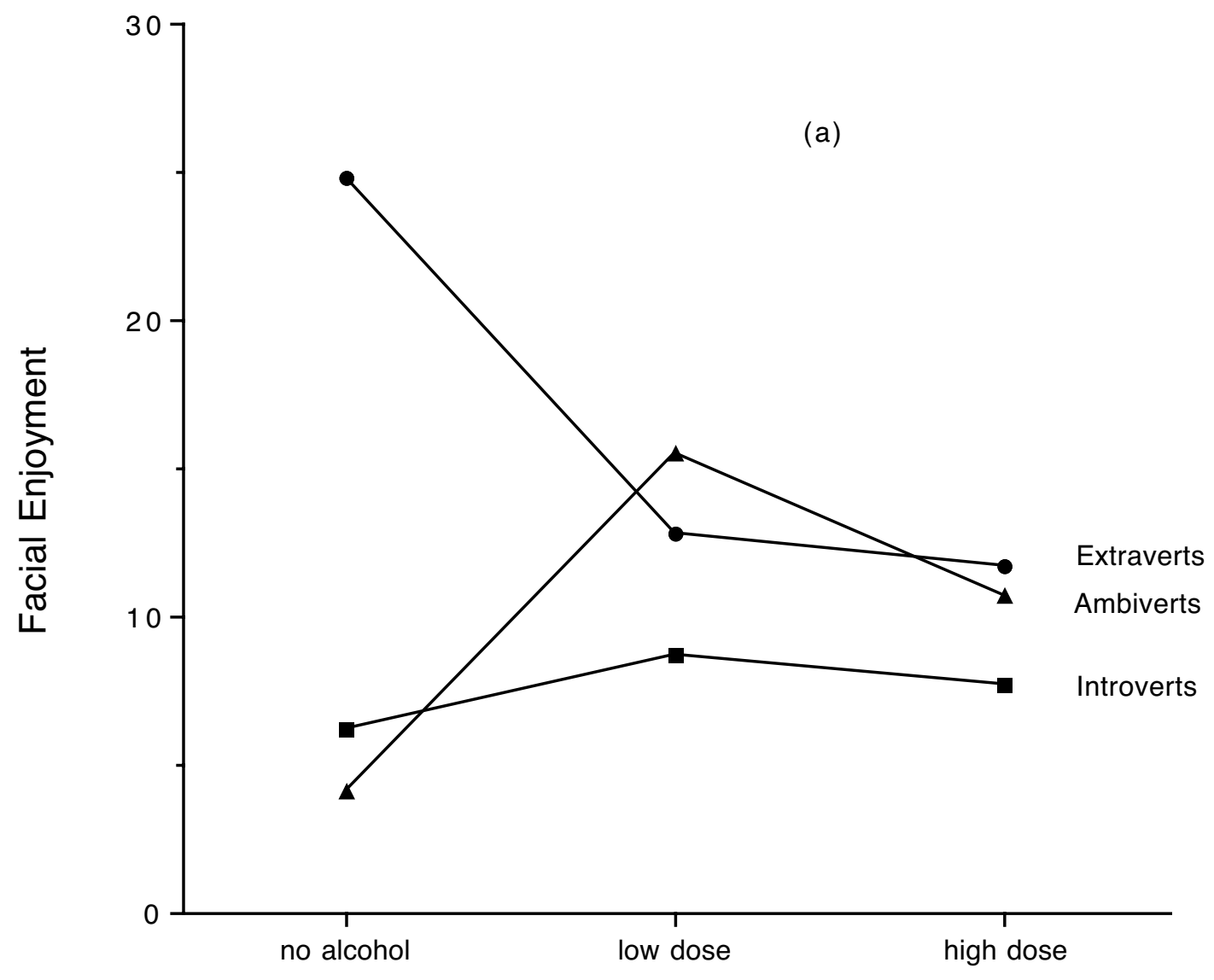

Experimental Groups

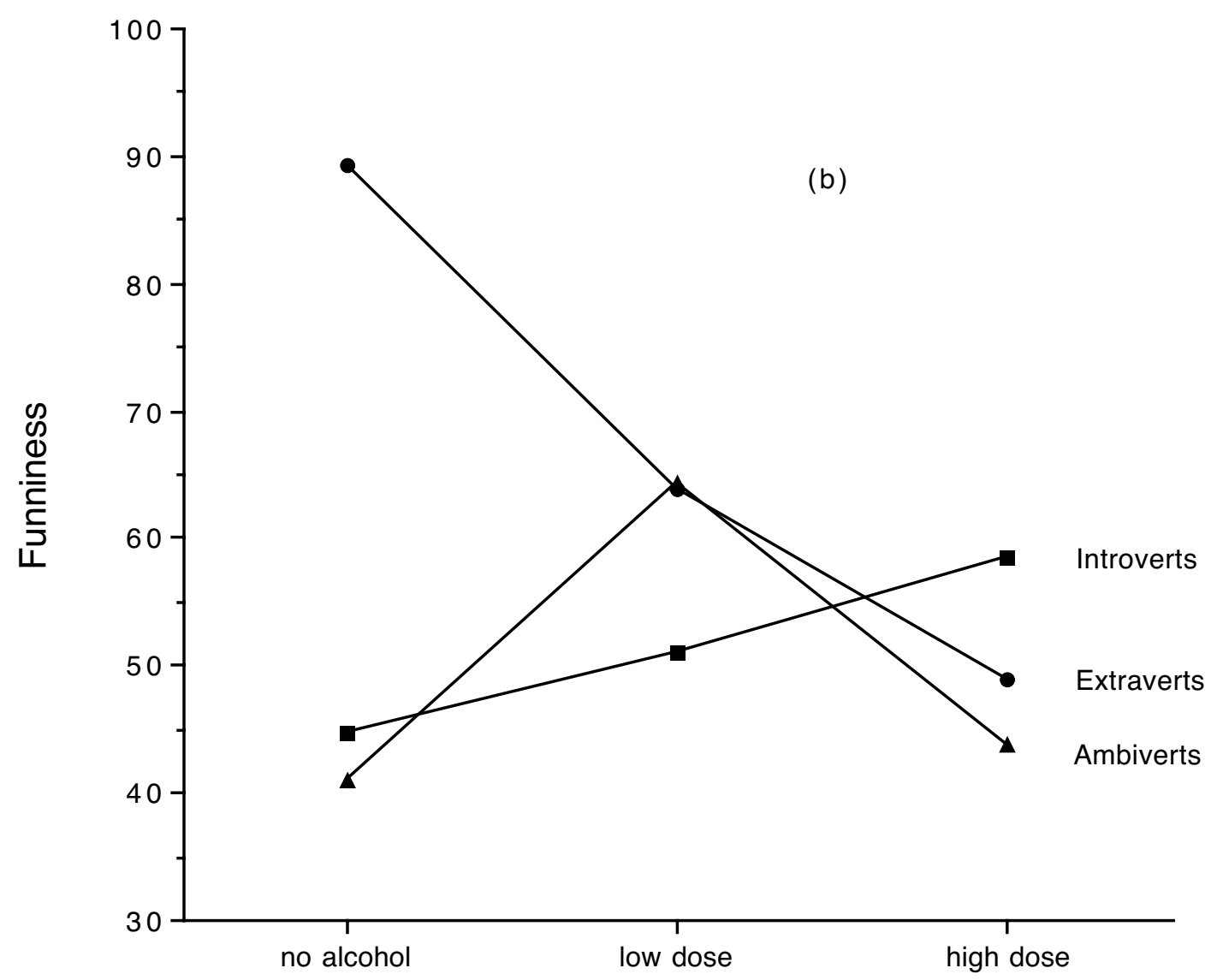

Experimental Groups 